\title{
CIRCUITOS ELÉCTRICOS: UNA APLICACIÓN DE UN MODELO DE ENSEÑANZA-APRENDIZAJE BASADO EN LAS IDEAS PREVIAS DE LOS ALUMNOS
}

\author{
VARELA NiETo, P., MANRIQUE de CAMPo, M.J. y FAVIERES MARTínez, A. \\ Instituto de Bachillerato «Rey Pastor».
}

\section{SUMMARY}

In this paper we wish to present the practical realization of the constructivist view of knowledge, carried out with an experimental type of methodology.

The phases of the work have been: the detection on preconcepts, the elaboration of the curriculum, the design of didactic materials and the evaluation of the results obtained.

The research has been carried out on the topic «electric current" at the level of 2 nd. year of B.U.P.

\section{INTRODUCCIÓN}

Los resultados de numerosas investigaciones realizadas en las últimas décadas acerca del modo como los estudiantes adquieren conocimientos ponen de manifiesto que, antes de iniciar un aprendizaje formal de la Ciencia, poseen unas ideas sobre las leyes que rigen el mundo que les rodea. En general estas ideas no concuerdan con el punto de vista científico.

Hay en la actualidad un gran interés por conocer cuáles son las ideas previas de los alumnos, ya que, según las modernas teorías sobre el aprendizaje, sólo se logra que éste sea significativo si se parte del conocimiento de estos preconceptos para construir, modificándolos, nuevos esquemas concep tuales (Ausubel 1976). Varios grupos de investigación están tratando de diseñar entrategias de instrucción en esta línea (Driver 1985, Nussbaum y Novick 1982, Osborne et al. 1985). Por ello, nuestro trabajo se sitúa en esta misma orientación y se ha centrado en los siguientes puntos:

- Detección de los preconceptos que poseen los alumnos sobre circuitos eléctricos.

- Diseño de un curriculo para conseguir un aprendizaje significativo.

- Evaluación de dicho aprendizaje.

\section{FUNDAMENTO TEÓRICO}

En las diferentes líneas de investigación que existen para el estudio de las ideas previas de los alumnos, estas re- ciben, según se consideren por los autores, diversas denominaciones tales como: esquemas conceptuales alternativos (Driver et al. 1983), ciencia de los niños (Os. borne et al. 1983), errores conceptuales (Gil y Carrascosa 1982), conceptos de Física y Química cotidianos (Andersson 1986).

En general todos to autores coinciden en que estas ideas previas se caracterizan por tener una cierta coherencia interna, a pesar de que los alumnos las usan de un modo aparentemente contradictorio, ser comunes a estudiantes de diferentes medios y edades, y estar tan fuertemente ar raigadas que son muy resistentes al cambio.

El punto de partida de nuestro trabajo ha sido la con* vicción de que los preconceptos que poseen nuestros alumnos en el campo investigado son similares a los detectados por Osborne, Shipstone, Tiberghien y otros, que se concretan esencialmente en este nivel en:

I) Idea de fuente-consumidor: muchos estudiantes piensan en la electricidad como en un fluído que se almacena en la bateria y se gasta en el funcionamiento de distintos aparatos. Por ello se utilizan indistintamente los términos «electricidad», «corriente», «energía eléctrica»o «potencia».

\section{II) Modelos de corriente en circuitos simples}

Al tratar de explicar como llega de la fuente al consumidor «lo que se gasta» los alumnos emplean diferentes modelos que, representados de forma esquemáti- 
ca, son los siguientes:

a) Modelo unipolar (fig. 1)

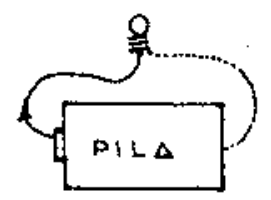

Este modelo presenta distintas variantes: el cable de retorno puede ser omitido o bien considerarse necesario, pero como elemento pasivo.

b) Modelo concurrente (fig. 2)

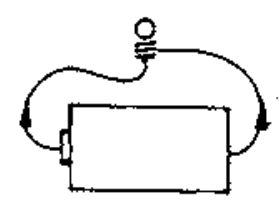

La corriente sale por los dos terminales de la batería y se consume en la bombilla.

c) Modelo de atenuación (fig. 3)

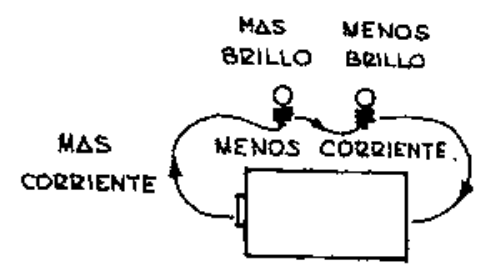

La corriente circula en una dirección alrededor del circuito, debilitándose graduaimente. Los últimos componentes recibirán menos y la primera lámpara brillará más que la segunda aunque ambas sean iguales.

d) Modelo de reparto (fig. 4)

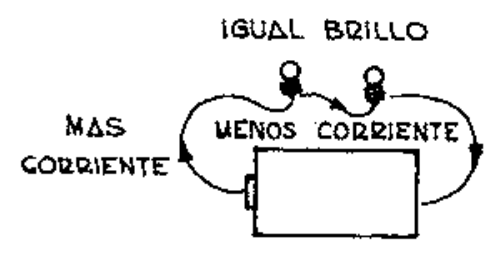

En este modelo, que es también no conservativo, la corriente se reparte entre los elementos del circuito. Las dos lámparas iguales brillarán to mismo.

III) Razonamiento secuencial: En los circuitos más complejos, que incluyen varios elementos se hacen más sutiles y responden a un análisis local del circuito (Closset, J. 1983). Por ejemplo, consideran que si se modi- fica un elemento no cambia la corriente que entra en él, sino que afecta solamente a la corriente que sale de él. En este modelo la corriente no se conserva.

Este tipo de razonamiento aparece en la enserianza se. cundaria por lo que algunos autores consideran que es debido a la secuencia en que se introducen los conceptos en este nivel.

IV) Potencial: En este punto es muy frecuente entre los alumnos la confusión entre corriente y voltaje (Rho. neck, 1983) y también la consideración de este último como una "consecuencia" de que la corriente circule y no como su «causa» (Cohen et al. 1982).

A partir de la detección de las ideas previas de nuestros alumnos hemos procedido a elaborar un material de trabajo siguiendo las pautas marcadas desde una perspectiva constructivista del aprendizaje (Driver 1986; Gil 1986). Estas pautas son:

i) Promover situaciones en que los alumnos expresen explicitamente sus ideas.

ii) Conseguir que los alumnos cuestionen sus puntos de vista, haciéndoles discutir en grupo, y emitan hipótesis acerca del comportamiento de determinados sistemas.

iii) Contrastar dichas hipótesis con los resuitados obtenidos experimentalmente.

iiii) Crear situaciones en que los alumnos apliquen $y$ reafirmen las nuevas ideas.

\section{FASES DEX TRABAJO}

\subsection{Detección de preconceptos}

Como hemos dicho anteriormente, la primera parte del trabajo ha consistido en confirmar la existencia en nues. tros alumnos de los conceptos previos detectados por los autores citados, en lo referente a circuitos elementales. Para elio hemos utilizado cuatro pruebas tipo test, diseñadas por dichos autores.

Incluimos como ejemplo una de las pruebas utilizadas para detectar modelos de corriente en un circuito ele. mental. (Fig. 5).

El problema del razonamiento secuencial no ha sido investigado debido a que nuestros alumnos están iniciando la Enseñanza Secundaria, y este punto responde a un análisis más complejo del circuito.

\subsection{Confección del currículo}

Una vez analizados los resultados de las pruebas realizadas, hemos ordenado los contenidos correspondien. tes a Energía Eléctrica, en este nivel, de acuerdo con el siguiente esquema: 
figura 5

Pruebas para la delección de modelos.

Una pita se conecta a ura bombilla como se muestra en el diagrama. La bombilla está luciendo.

¿Con qué diagrama piensas que se describe mejor la corriente eléctrica en los cables?

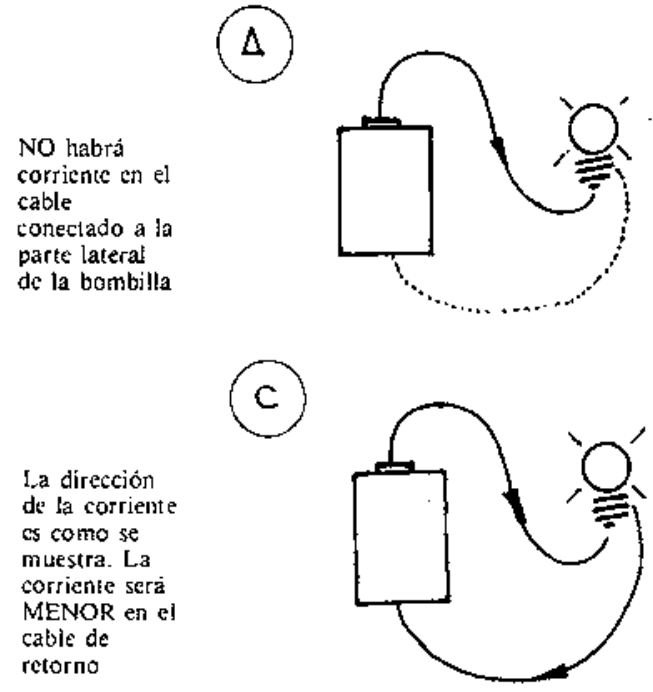

\section{ENERGIA ELÉCTRICA}

1. Energia eléctrica: transformaciones.

2. ¿Cóno se reparte la energía en un circuito?

3. ¿Quién transporta la Energía Eléctrica?

4. Modelos de corriente. Intensidad.

5. ¿Qué significa voltaje o diferencia de potencial?

6. ¿De qué depende la intensidad de la corriente eléctrica a través de un conductor metálico?

6.1. Ley de Ohm. Resistencia de un conductor metálico. 6.2. Circuitos en serie y paralelo. Aplicaciones.

\section{La electricidad en casa.}

El desarrollo de este currículo responde a las siguientes ideas básicas:

i) Iniciar el tema partiendo de la conservación de la energía en un circuito para encauzar dentro de un punto de vista científico el preconcepto que los alumnos tienen de fuente-consumidor, y también para eludir el problema del estudio del campo eléctrico, que nos parece demasiado abstracto para este nivel.

ii) Introducir la corriente eléctrica como una explicación del modo en que se transporta la energia aprovechando este planteamiento energético para definir el concepto de voltaje como la energía gastada por unidad de carga que atraviesa un receptor (Hartel, 1982).

iii) Promover la inclusión de las ideas adquiridas en el aula dentro del mundo cotidiano de los alumnos.

\subsection{Metodología}

Para poner en práctica el modelo de enseñanza-
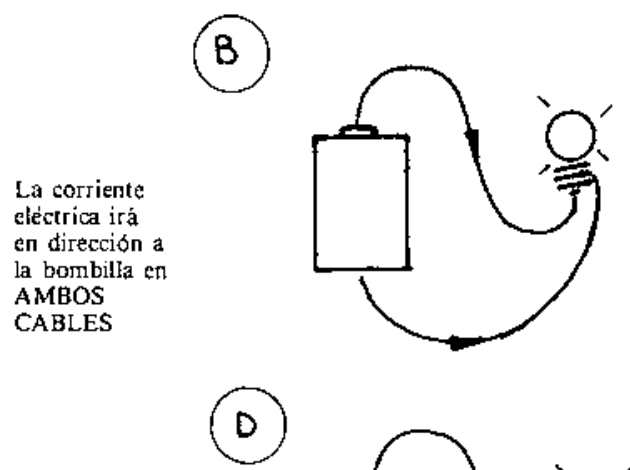

La dirección

de la corriente es como se

muestra. 1.a

corriente será

la misma en

anbos cables

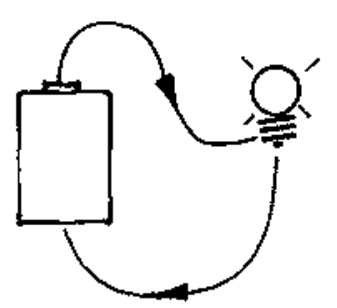

aprendizaje constructivista hemos elaborado unas fichas sobre las cuales trabajan los alumnos, persiguien. do estos objetivos:

- Hacer reflexionar a los alumnos sobre una cuestión para, a continuación, discutir sus hipótesis en grupos de cuatro. El resultado de la discusión queda anotado en la ficha, con lo cual se pretende que tomen conciencia de sus ideas previas y de las de sus compañeros.

- Realizar el montaje propuesto en la cuestion incluyendo en él aparatos de medida y anotar sus resultados.

Tratar de explicar los resultados experimentales obtenidos, de acuerdo con sus hipótesis y, en caso de conflicto, elaborar otras nuevas que expliquen satisfactoriamente los hechos.

- Afianzar las nuevas ideas mediante la resolución de otras cuestiones planteadas sobre el mismo problema.

- Proporcionar oportunidades a los estudiantes para integrar estos conocimientos en su ambiente diario.

Como aclaración de todo lo expuesto, incluimos dos fichas que tratan de afianzar el modelo conservativo de corriente (Fig. 6).

\section{RESULTADOS Y CONCLUSIONES}

La experiencia se ha realizado con tres grupos de alumnos de $2^{\circ}$ de BUP del IB Rey Pastor de Madrid y ha tenido una duración de cinco semanas, al final de las cuales se han repetido las pruebas iniciales, para eva. luar si se ha producido un cambio conceptual. 
figura 6

Dos modelos de fichas para afianzar el modelo conservativo de la corriente.

Vamas a Interpreter el circuito de lo flgura o peuarda con nuestro modelo de corriente.

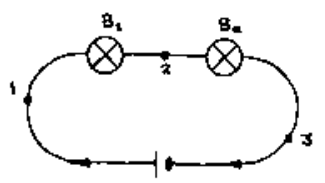

¿Como debe ser la Intensidad in

unyor en el punto 1 que gn el punto 2 , $\gamma$ en

3 moyas aue en al 3.

Igual en 2,2 y 3

La bonbilia $\mathrm{B}_{2}$ brilla eás que la basoblila $\theta_{2}$ Lag dos bombilless todilian igud

Aezona tus contestaejoncs.

Pare comprobur vuestras conclussones vais a mentor ol efrcutio y modir le Intensidpa en fido sunto

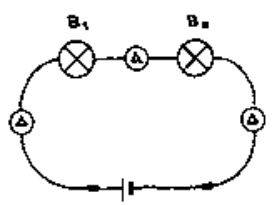

Anote lo que mercan las amperimetros y a 10 viste de las resultedos revisa tus prediccio nos.

Los primeros resultados obtenidos sobre detección de preconceptos indican el modo en que los alumnos conectarian una pila a una bombilla para que ésta luzca. (Fig. 7).

\section{figura 7}

Formas de conexión de una pila a una bombilla.

\section{$8.8 \%$}

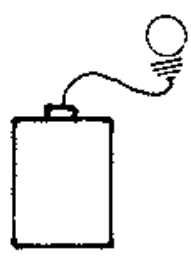

$2.6 \%$

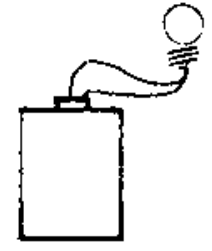

$26.6 \%$

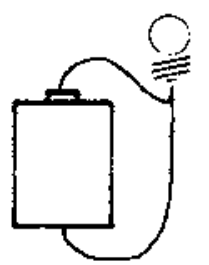

$62 \%$

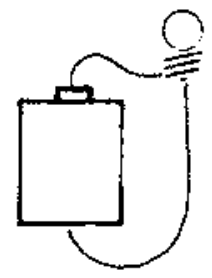

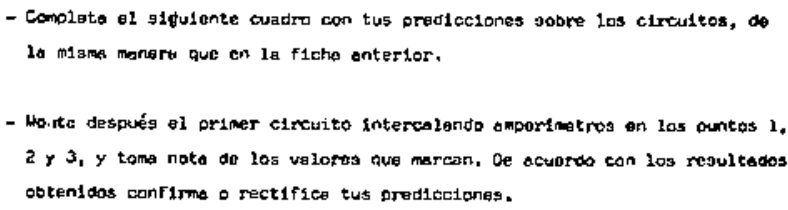

- Complate el gigulente cuadru con tus pradeciones vobre las cirtules, de la misa manore que en la f1che anterlor.

- Ho rte después el primer c1reuito intercalendo amporimakros on las ountos 1 . 2 y 3, y toma nota do las valores hue marcan, de acuoros con los rejul tedos ootenidos canfirme o rectifice tus gredicciones.

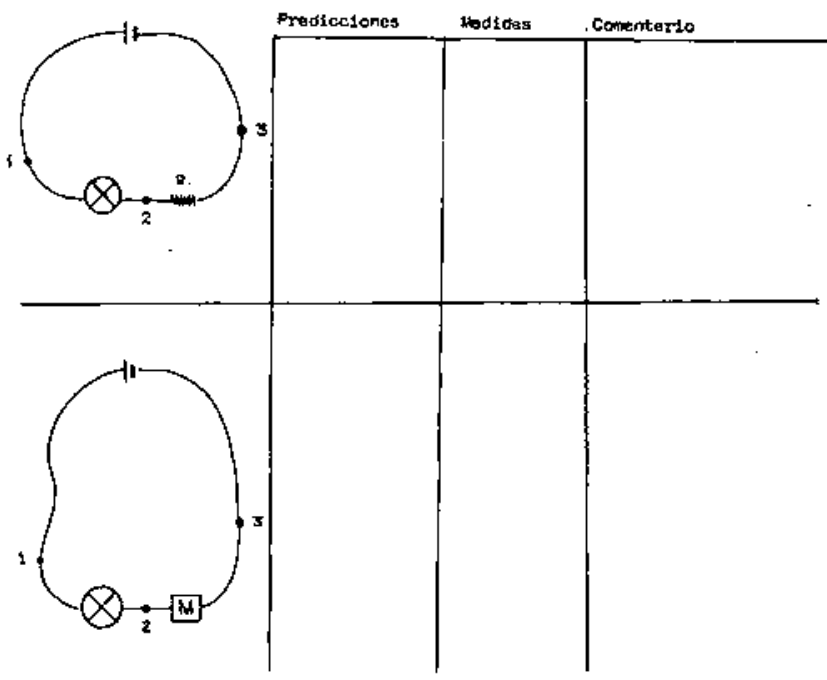

Puede resultar sorprendente que en este nivel de enseñanza aún exista un porcentaje significativo de alumnos que no es capaz de realizar el montaje correcto. Para intentar solucionarlo procedimos a una entrevis- ta con cada alumno en el laboratorio, dándole el material necesario para que realizaran la conexión adecuada. Después de la misma prácticamente todos los alumnos superaron el problema.

Respecto de los modelos de corriente adoptados por los alumnos se han conseguido los siguientes resultados globales (Tabla I).

Quizás lo más significativo de estos resultados sea que los alumnos han abandonado la idea de fuenteconsumidor en sus distintas versiones.

Con respecto al concepto de diferencia de potencial, los resultados son los que recoge la Tabla II.

Tabla I

$\%$ Alumnos que optan por cada modelo

\begin{tabular}{|l|l|l|l|l|l|}
\hline MODELO & CIENTINCO & bONCURROHE & ATENUACION & REPARTO & OTROS \\
\hline INICIAL & 40.74 & 31.48 & 14.11 & 9.25 & 7.40 \\
\hline FINAL & 84.26 & 0 & 3.73 & 2.80 & 8.41 \\
\hline
\end{tabular}


Tabla II

\begin{tabular}{|c|c|c|}
\hline & $\begin{array}{c}\text { DIFERENCIAN } \\
\text { VOLTAJE dR INTENSIDAD }\end{array}$ & $\begin{array}{c}\text { APLICAN CORRECTAUENTE } \\
\text { MMBOS CONCEPTOS }\end{array}$ \\
\hline$I N I C I \Delta L$ & 14.9 & 0 \\
\hline FINAL & 25.3 & 24.5 \\
\hline
\end{tabular}

Inicialmente, un gran porcentaje de alumnos no diferenciaban entre corriente y voltaje, y ninguno de ellos era capaz de aplicar estos conceptos correctamente en circuitos eléctricos. Al finalizar el aprendizaje los alumnos que eran capaces de diferenciar ambos conceptos también sabían aplicarlos correctamente en circuitos tanto abiertos como cerrados, aunque este porcentaje no fuera elevado debido a la dificultad del tema.

Por otra parte, nos interesó comparar el nivel de conocimientos adquirido por los alumnos que habían realizado la experiencia con el de otros alumnos del mismo centro. Para ello se ha propuesto a todos una prueba clásica de contenido (diferente a las diseñadas para medir el cambio conceptual en los alumnos «experimen- tales») que ha dado un resultado superior en el grupo experimental con un nivel de significación del $95 \%$.

A pesar del aparente éxito de este sistema de trabajo, los resultados no son concluyentes, pues, según muchos autores (McDermott 1984, Osborne 1985, Tiberghien 1983), las ideas originales de los alumnos vuelven a reimplantarse pasado cierto tiempo, y persisten incluso en los niveles de enseñanza superior (Viennot 1979). Debido a esto es imprescindible seguir la investigación en este campo comprobando si se mantiene el cambio conceptual, para lo cual tendremos que pasar las mismas pruebas al cabo de seis meses de finalizada la instrucción y continuar la misma línea de investigación en todos los niveles.

\section{REFERENCIAS BIBLIOGRAFICAS}

ANDERSSON, B., 1986, The experiential gestalt of causation: a common core to pupil's preconceptions in Science. European Journal of Science Education, Vol. 8, $\mathrm{n}^{\circ}$ 2, pp. 155-171.

AUSUBEL, D., NOVAK, J., HANESIAN, H., 1976, Psicología educativa. Un punto de vista cognoscitivo. (Trillas: Méjico).

COHEN, R,, EYLON, B., GANIEL, U., 1983, Potencial difference and current in simple electric circuits: a study of student's concepts. American Journal of Physics, 51 (5), pp. 407-412.

CLOSSET, J., 1983, Le raisonnement sequential en Electrocinetique. Atelier Internacional d'eté: Recherche en Didactique de la Physique. La Londe les Maures.
DRIVER, R., ERICKSON, G., 1983, Theories in action: some theorical and empirical issues in the study of student's conceptual frameworks in Science. Studies in Science Education, Vol. 10, pp. 37-60.

DRIVER, R., GUESNE, E., TIBERGHIEN, A., 1985, Children's ideas in Science. (Open University Press: Milton Keynes, England).

DRIVER, R., 1986, Psicología cognoscitiva y esquemas conceptuales de los alumnos. Enseñanza de las ciencias. Vol. 4, (1), pp. 3-15.

GIL, D., CARRASCOSA, J., 1982, Los errores conceptuales en la enseñanza de la Física, 1as. Jornadas de Investigación Didáctica en Física y Química. Valencia. 
GIL, D., Bases teóricas de un modelo de enseñanza/aprendizaje de las Ciencias. Simposio sobre Psicología del aprendizaje y desarrollo curricular, Oviedo. pp. 127-134.

HÄRTEL, H., 1982, The electric circuits as a system: a new approach. European Journal of Science Education, Vol. 4 (1), pp. 45-55.

McDERMOTT, L., 1984, Research on conceptual understanding in mechanics. Physics Today, July, pp. 24-32.

NUSSBAUM, J., NOVICK, S., 1982, Alternative framework, conceptual conflict and acommodation: towards a principled teaching strategy. Instructional Science. Vol. It, pp. 183-200.

OSBORNE, R., BELL, F., GILBERT, J., 1983, Science teaching and children's views of world. European Journal of Science Education. Vol. 5 (1), pp. 1-14.

OSBORNE, R., FREYBERG, P., 1985, Learning in Science. (Hainemann England).

OSBORNE, R. et al, Learning in Science Project. Working papers $n^{\circ} 25,207,209$. (University of Waikato. Hamilton. N.Z.).
RHÖNECK, C., 1983, Semantics Structures describing the electric circuit before and after instruction. International Summer Work-shop: Research on Physics Education. La Londe les Maures. Francia.

SHIPSTONE, D., 1984, A study of children's understanding of electricity in simple D.C. circuits. European Journal of Science Education. Vol. 6, (2), pp. 185-198.

TIBERGHIEN, A., DELACOTTE, G., 1976, Manipulations et représentations de circuits élèctriques simples par des enfarts de 7 a 12 ans. Revista francesa de pedagogía. $\mathrm{N}^{\circ}$ 34 , pp. $32-44$.

TIBERGHIEN, A., 1983, Revue critique sur les recherches visant a elucider le sens des notions de circuits élèctriques pour les èleves de 8 a 20 ans. Atelier International d'eté: Recherche en Didactique de la Physique. La Londe les Maures. France.

VIENNOT, L., 1979, Spontaneus Reasoning in Elementary Dynamics. European Journal of Science Education. Vol. 1, (2), pp. 205-221. 